\title{
Coronal versus photospheric abundances of stars with different activity levels ${ }^{\star}$
}

\author{
J. Sanz-Forcada ${ }^{1}$, F. Favata ${ }^{2}$, and G. Micela ${ }^{1}$ \\ 1 INAF - Osservatorio Astronomico di Palermo G. S. Vaiana, Piazza del Parlamento, 1, Palermo, 90134, Italy \\ 2 Astrophysics Division - Research and Science Support Department of ESA, ESTEC, Postbus 299, 2200 AG Noordwijk, \\ The Netherlands
}

Received 7 October 2003 / Accepted 6 November 2003

\begin{abstract}
We report a detailed analysis of the coronal abundance of 4 stars with varying levels of activity and with accurately known photospheric abundances. The coronal abundance is determined here using a line flux analysis and a full determination of the differential emission measure. Photospheric abundance values are taken from the literature. Previous coronal abundance determinations have generally been compared to solar photospheric abundances; from this a number of general properties have been inferred, such as the presence of a coronal metal depletion with an inverse First Ionization Potential correlated with activity level. Here we show that, when coronal abundances are compared with real photospheric values for the individual stars, the resulting pattern can be very different. Also, we present evidence that, in some cases, the coronal metal abundance may not be uniform in the corona; in particular it can vary with the temperature of the emitting plasma.
\end{abstract}

Key words. stars: coronae - stars: abundances - stars: late-type - X-rays: stars - line: identification

\section{Introduction}

Following the initial results from the ASCA-SIS instrument (e.g. White et al. 1994; Drake 1996), which showed that the low-resolution X-ray spectra of active coronal sources were often best fit with a low metal abundance (typically about 0.3 the solar photospheric abundance), the issue of the metal abundance of the coronal plasma has become one of the main areas of interest for X-ray spectroscopy of active stars. Due to the limited spectral resolution of the ASCA spectra, the determination of abundance for elements other than $\mathrm{Fe}$ was often not accurate (Favata \& Micela 2003). The availability of the XMM-Newton and Chandra high-resolution X-ray spectra of coronal sources has allowed observing individual, resolved spectral lines of a number of elements in the corona of active stars, and thus to address the issue of the abundance of a number of elements. Unfortunately up to now the results have not been as conclusive as initially expected. One reason is that often the same data analyzed with different approaches result in significantly different values for the element abundances (see Favata \& Micela 2003, for a number of examples).

Send offprint requests to: J. Sanz-Forcada, e-mail: jsanz@astropa.unipa.it

* Tables 4-7 are only available in electronic form at the CDS via anonymous ftp to cdsarc.u-strasbg.fr $(130.79 .128 .5)$ or via http://cdsweb.u-strasbg.fr/cgi-bin/qcat?J/A+A/416/281
One of the issues most often addressed by coronal abundance studies is whether a bias of the First Ionization Potential (FIP) of the element is present. Such an "FIP effect" is found in the solar corona, where the abundance of elements with low FIP ( $<10 \mathrm{eV}$; e.g. $\mathrm{Mg}, \mathrm{Si}, \mathrm{Fe})$ is observed to be enhanced with respect to their photospheric values, while elements with high FIP ( $\gtrsim 10 \mathrm{eV}$; e.g. O, Ne, Ar) remain at abundances similar to the photospheric values. Such a behavior is observed in the upper solar atmosphere at temperatures higher than $\sim 1 \mathrm{MK}$, both in the non-active regions and in full-disk spectra (Laming et al. 1995; Feldman \& Laming 2000). EUVE observations showed the same FIP bias to be present in stars with low activity levels, such as $\alpha$ Cen AB (G2V+K1V, Drake et al. 1997), and possibly (only limited knowledge of their photospheric abundance was available at that time) $\xi$ Boo A $(\mathrm{G} 8 \mathrm{~V}$, Laming \& Drake 1999) and $\epsilon$ Eri (K2V, Laming et al. 1996). EUVE spectra of more active stars did not show any FIP effect, with however a rather low coronal Fe abundance with respect to the solar photosphere, which suggested some metal depletion in the corona of the active stars. Feldman \& Laming (2000) review some of the results on solar and stellar coronal abundances previous to Chandra and XMM-Newton.

The new generation of X-ray satellites XMM-Newton and Chandra offers access to high resolution spectra in a wavelength region where numerous spectral lines from different elements are formed at coronal temperatures. This permits one to measure the fluxes of individual lines, in principle allowing a 
Table 1. Observation log.

\begin{tabular}{lccccrcrrrr}
\hline \hline Object & HD \# & SpT & Instrument & $\lambda \lambda(\AA)$ & $t_{\text {exp }}(\mathrm{ks})$ & Date & $d(\mathrm{pc})$ & $L_{\mathrm{X}}$ & $L_{\mathrm{X}} / L_{\text {bol }}$ & $L_{\mathrm{X}}^{\prime}$ \\
\hline Procyon & 61421 & F5IV & Chandra/LETG & $3-175$ & 69.6 & 6 Nov. 1999 & 3.50 & $8.89 \mathrm{E} 27$ & $3.39 \mathrm{E}-7$ & $9.41 \mathrm{E} 26$ \\
Procyon & 61421 & F5IV & Chandra/LETG & $3-175$ & 69.7 & 7 Nov. 1999 & “ & “ & “ & “ \\
$\epsilon$ Eri & 22049 & K2V & Chandra/LETG & $3-175$ & 105.3 & 21 Mar. 2001 & 3.22 & $1.79 \mathrm{E} 28$ & $1.42 \mathrm{E}-5$ & $9.65 \mathrm{E} 27$ \\
$\lambda$ And & 222107 & G8III/? & Chandra/HETG & $1.7-27$ & 81.9 & 17 Dec. 1999 & 25.8 & $2.74 \mathrm{E} 30$ & $3.25 \mathrm{E}-5$ & $1.73 \mathrm{E} 30$ \\
V851 Cen & 119285 & K2IV-III/? & XMM/RGS & $4-38$ & 29.2 & 7 Mar. 2003 & 76.2 & $6.46 \mathrm{E} 30$ & $3.01 \mathrm{E}-4$ & $4.47 \mathrm{E} 30$ \\
\hline
\end{tabular}

Note: Distances $(d)$ from Perryman et al. (1997). $L_{\mathrm{X}}\left(\mathrm{erg} \mathrm{s}^{-1}\right)$ in the range 5-100 $\AA(0.12-2.4 \mathrm{keV})$, the range covered by the ROSAT/PSPC. $L_{\mathrm{X}}^{\prime}\left(\mathrm{erg} \mathrm{s}^{-1}\right)$ in the range 6-20 $\AA(0.62-2.1 \mathrm{keV})$, common to RGS, HETGS and LETGS.

much better determination of the coronal thermal structure and abundances. Initial results on coronal abundances determined from data from the two observatories have been reviewed by Drake (2002) and Favata \& Micela (2003). These new results seem to point towards a scenario with stars with low activity levels showing, when their coronal and photospheric abundances are compared, a solar-like FIP effect (case of $\alpha$ Cen, Raassen et al. 2003) or no FIP bias (for Procyon, Raassen et al. 2002). Active stars (like HR 1099, II Peg, AR Lac or AB Dor), with a quite different coronal thermal structure, dominated by material emitting at temperatures of $\sim 10 \mathrm{MK}$, show a pattern characterized by a general depletion of elements with low FIP (when they are compared with solar photospheric values) while the elements with high FIP remain at solar (or sometimes higher) photospheric values (Drake et al. 2001; Brinkman et al. 2001; Huenemoerder et al. 2001, 2003; Sanz-Forcada et al. 2003b) although photospheric abundances of elements other than Fe are not known for HR 1099. Audard et al. (2003) analyzed XMM-Newton observations of 5 active binary systems, concluding that low-FIP elements are subject to greater depletion in more active stars, while high-FIP elements remain at similar levels (although photospheric abundances were available only for one of the objects in their sample, namely $\lambda$ And). In general, the comparison of stellar coronal abundances with solar photospheric values is of little use, given the wide range of abundances found among late type stars (e.g. Cayrel de Strobel et al. 2001). Most of the stars for which coronal abundances have been determined have no published photospheric abundances, so that a true test of FIPrelated effects cannot be done. This is especially true for active stars, for which the large rotational velocity complicates the measurements of photospheric abundances. In most cases the available photospheric abundances are limited to Fe (e.g. Randich et al. 1993), and even these in many cases are likely to be affected by systematic errors. Moreover, different methods to determine the distribution of material with temperature in the corona (the so called "emission measure distribution", EMD) sometimes produce contradictory results, depending on the analysis technique employed (Favata \& Micela 2003; Sanz-Forcada et al. 2003b.

In the present work we determine the coronal thermal structure and abundances of four stars with different activity levels (in terms of $L_{\mathrm{X}} / L_{\text {bol }}$ ) observed with relatively high statistics with Chandra and XMM-Newton (see Table 1), and for which there are accurate photospheric abundance measurements available, to explore the relation between activity levels and coronal relative abundance patterns. The chosen stars are Procyon (one of the stars with lowest $L_{\mathrm{X}} / L_{\text {bol }}$ values), the intermediate activity star $\epsilon$ Eri, the long-period active binary $\lambda$ And and the active binary V851 Cen. These four objects are among the few stars for which both high-resolution coronal spectra with good statistics and measurements of photospheric abundances for several elements are available.

The observations are described in Sect. 2, a brief description of the analysis techniques is presented in Sect. 3. The results for each star are discussed in Sect. 4 , followed by the discussion and conclusions in Sect. 5.

\section{Observations}

We have analyzed observations conducted using different $\mathrm{X}$-ray high-resolution spectrographs; however, the spectral lines observed in the different spectra are formed in very similar temperature ranges $(\log T[\mathrm{~K}] \sim 5.5-7.8)$, hence yielding information of the same region of the corona (see Sect. 3). The X-ray luminosity of the targets has been calculated by modeling the observed spectra, as listed in Table 1.

\subsection{V851 Cen}

An XMM-Newton observation of V851 Cen (P.I. F. Favata) was executed in March 2003 (Table 1). XMM-Newton carries out simultaneous observation with the EPIC (European Imaging Photon Camera) PN and MOS detectors (sensitivity range $0.15-15 \mathrm{keV}$ and $0.2-10 \mathrm{keV}$ respectively), and with the RGS (Reflection Grating Spectrometer, den Herder et al. 2001) $(\lambda \lambda \sim 6-38 \AA, \lambda / \Delta \lambda \sim 100-500)$. The RGS data have been reduced employing the standard SAS (Science Analysis Software) version 5.4.1 package, removing in the RGS spectra the time intervals when the background was higher than $0.4 \mathrm{cts} / \mathrm{s}$ in CCD \#9, to ensure a "clean" spectrum. Light curves were obtained by selecting a circle centered on the source in the EPIC-pn and EPIC-MOS images, and subtracting the background count rate taken from a nearby area. The light curve shows a slow decline ( 20\%) of the source flux (Fig. 1). High resolution spectra corresponding to the first and second orders of the RGS have been used to measure the line fluxes simultaneously, improving the quality of the measurements. Fluxes measured for second order lines are in good agreement with those in the first order (Sanz-Forcada et al. 2003b), hence they 

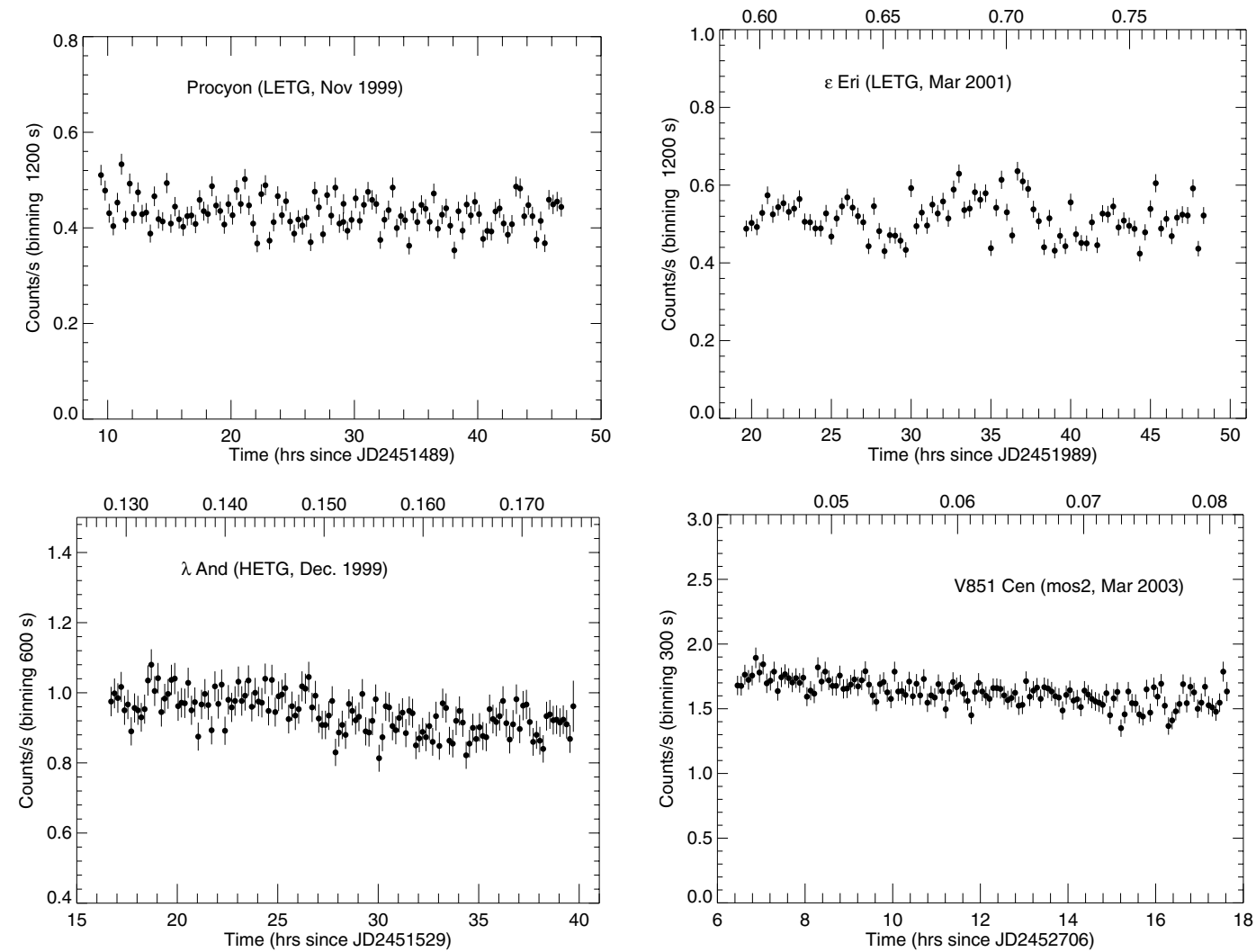

Fig. 1. Light curves of Procyon (LETGS), $\epsilon$ Eri (LETGS), $\lambda$ And (HETGS) and V851 Cen (XMM/MOS 2) with 1- $\sigma$ error bars. The upper axis reports the photometric phase ( $\epsilon$ Eri) and orbital phase ( $\lambda$ And and V851 Cen, with $T_{0}$ corresponding to secondary star located behind the primary star).

can be used to improve the quality of the measurements due to their better spectral resolution.

\section{2. $\lambda$ And}

An observation with the Chandra High Energy Transmission Grating Spectrograph (HETG, Weisskopf et al. 2002) of $\lambda$ And was retrieved from the Chandra archive (Table 1). The HETGS is made of two gratings, HEG (High Energy Grating, $\lambda \lambda \sim 1$.715, $\lambda / \Delta \lambda \sim 65-1070$ ), and MEG (Medium Energy Grating $\lambda \lambda \sim$ 3-27, $\lambda / \Delta \lambda \sim$ 80-970) operating simultaneously. Standard reduction tasks from the CIAO v2.3 package have been employed in the reduction of data and the extraction of the HEG and MEG spectra. The light curve of $\lambda$ And, obtained from the MEG and HEG first order spectra, shows no significant events, with variations of less than $20 \%$ of the total flux (Fig. 1).

\subsection{Procyon and $\epsilon$ Eri}

Observations with the Chandra Low Energy Transmission Grating Spectrograph (LETGS, $\lambda \lambda \sim 3-175, \lambda / \Delta \lambda \sim 60$ 1000 Weisskopf et al. 2002) and the High Resolution Camera (HRC-S) of Procyon and $\epsilon$ Eri were retrieved from the Chandra archive as listed in Table 1. Data were reduced using standard reduction tasks present in the CIAO v2.3 package. The positive and negative spectral orders were summed for the flux measurements, although at long wavelengths ( $~ 240 \AA)$ separate measurements were made in the plus and minus spectra due to problems with wavelength calibration or detector gaps. Lines formed in the first dispersion order but contaminated with contribution from higher dispersion orders were not employed in the analysis. The two observations available for Procyon were summed to achieve better statistics, resulting in an exposure time of $139 \mathrm{ks}$. Light curves were obtained from the LEG spectra (first and higher orders) of Procyon and $\epsilon$ Eri as shown in Fig. 1.

\section{Data analysis}

X-ray spectra from stellar coronae have numerous lines from different elements formed at different temperatures. This variety of temperatures in the lines formation makes it necessary to determine in detail the thermal structure of the target star, commonly parameterized through the emission measure distribution (EMD) as a function of temperature, defined as $E M(T)=\int_{\Delta T} N_{\mathrm{H}} N_{\mathrm{e}} \mathrm{d} V\left[\mathrm{~cm}^{-3}\right]$. In the present work we apply a line-based method to reconstruct the EMD of the stellar coronae (this method is preferred to the global fitting techniques, see Sanz-Forcada et al. 2003b), with a step in temperature of $0.1 \mathrm{dex}$, the same as employed in the Astrophysical Plasma Emission Database (APED v1.3, Smith et al. 2001), which is used for comparison of the observed with the predicted line fluxes as explained below. 

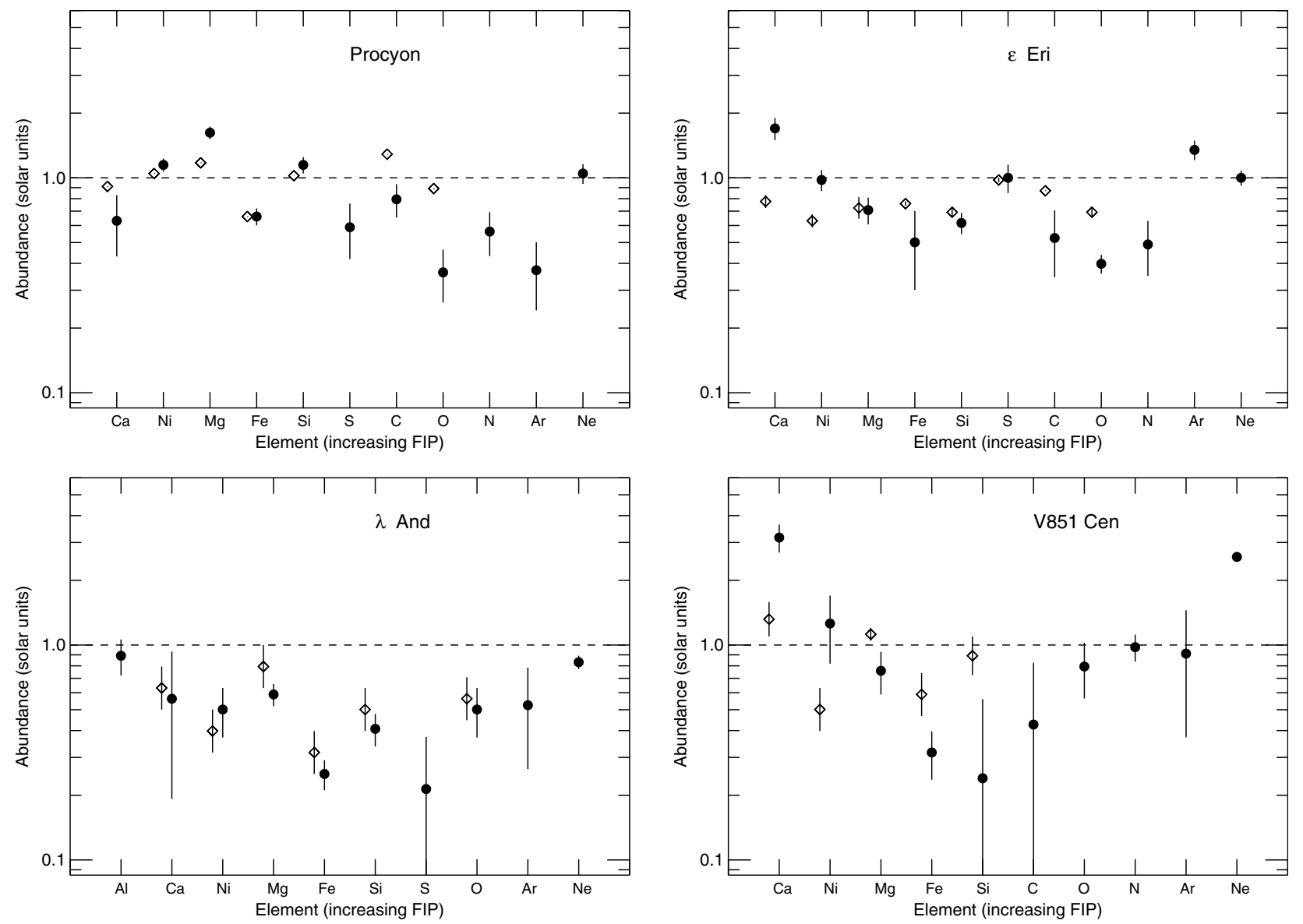

Fig. 2. Element abundances in the corona of Procyon, $\epsilon$ Eri, $\lambda$ And and V851 Cen (filled circles) with respect to solar photospheric values. Open diamonds represent the stellar photospheric abundances (see text). A dashed line indicates the adopted solar photospheric abundance (Anders \& Grevesse 1989).

The EMD reconstruction was conducted by measuring the fluxes from spectral lines present in the high resolution X-ray spectra, following Sanz-Forcada et al. (2003b). The Interactive Spectral Interpretation System (ISIS, Houck \& Denicola 2000) software package, provided by the MIT/CXC, was employed to measure the line fluxes, through convolution of the spectral response. These measured line fluxes were then corrected for interstellar medium absorption (ISM) (although such correction is important only for very few lines). Theoretical fluxes can be predicted by assuming an initial EMD which is combined with the emissivity functions of the different spectral lines. These fluxes are then compared with the observed fluxes, and the EMD is changed to determine a solution that shows a better agreement between predicted and observed fluxes. An iterative process, as explained in Sanz-Forcada et al. (2003b), results in the "best" solution for both the EMD and the abundance pattern. Although this solution is not unique, the use of a large number of spectral lines with a good temperature coverage constrains the interval of possible solutions to a small range of values around the solution proposed. Error bars for the EMD can be calculated with a Monte Carlo method, consisting of the variation of the observed fluxes by $\pm 1 \sigma$ and the determination of the best solution among 1000 possible variations around the initial EMD solution. Such an exercise is performed for 1000 sets of possible line fluxes, providing error bars for the solution. The abundances of the different elements are determined during the calculation of the EMD, with error bars resulting from the quadratic summation of the errors on the measurement of observed fluxes $\left(\Delta F_{\text {obs }} / F_{\text {obs }}\right)$, and the dispersion of the predicted-to-observed line ratios $\left[\left(F_{\text {obs }}-F_{\text {pred }}\right) / F_{\text {obs }}\right]$ of lines of the same element (the latter permits us to evaluate indirectly the errors related to atomic models). The abundances of the elements are determined relative to $\mathrm{Fe}$, with the $[\mathrm{Fe} / \mathrm{H}]$ abundance derived afterwards from the best fit to the global spectrum for the given EMD and abundances pattern. The coronal abundances determined, with their photospheric counterparts are shown in Figs. 2 and 3, and are listed in Table 2. EMD distributions (Table 3) are shown in Fig. 4. The line fluxes measured in the four targets are listed in Tables $4-7^{1}$.

\section{Results}

\subsection{Procyon}

Procyon ( $\alpha$ CMi A, HR 2943, HD 61421) is a well-studied nearby F4IV star (3.497 pc Perryman et al. 1997). A rotational period of $\sim 9.1 \mathrm{~d}$ can be deduced from $v \sin i=6.1 \mathrm{~km} \mathrm{~s}^{-1}$ (de Medeiros \& Mayor 1999) and $i=32^{\circ}$ (Irwin et al. 1992).

1 These tables are only available in electronic form at the CDS. 

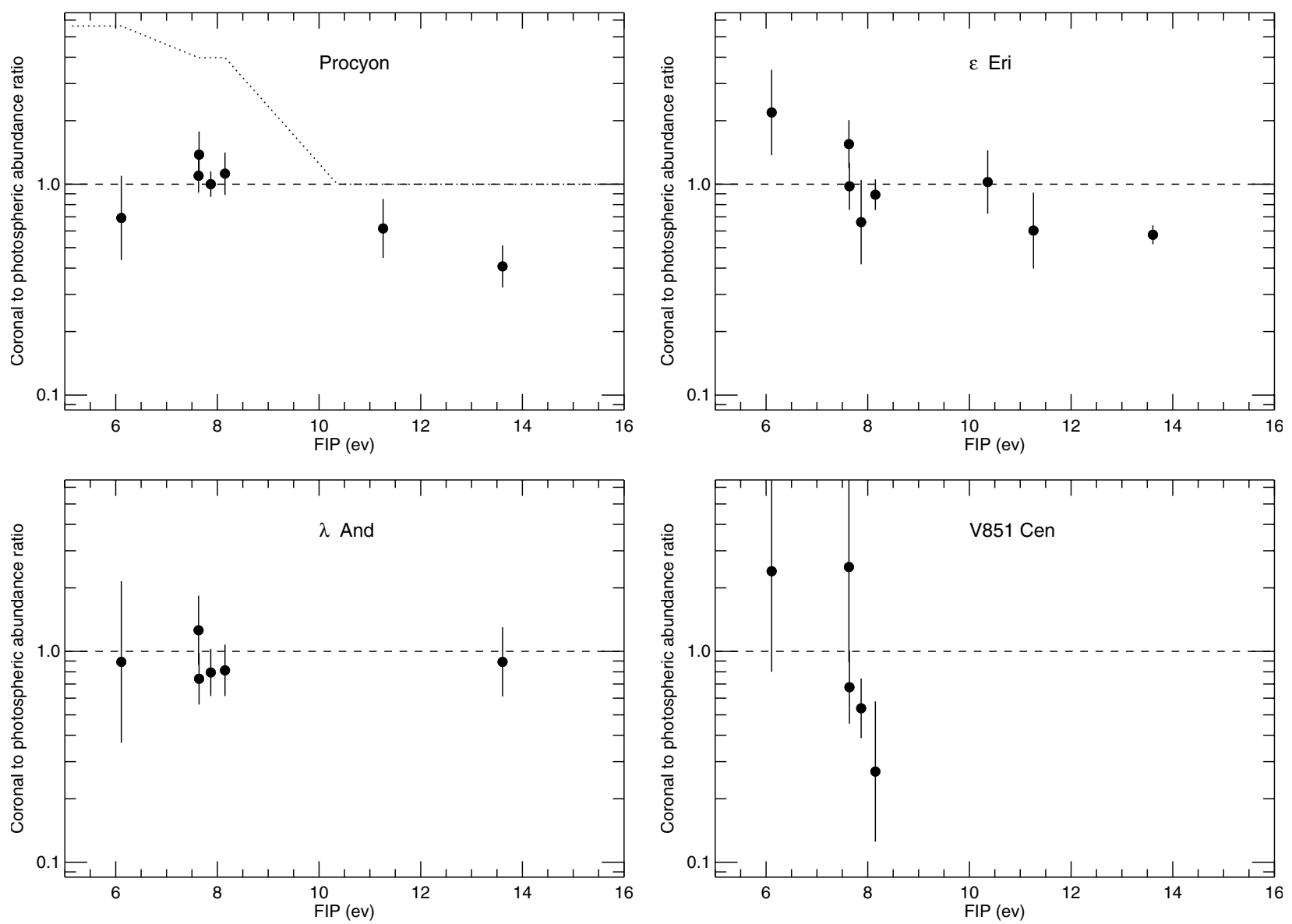

Fig. 3. Coronal to photospheric abundance ratio for Procyon, $\epsilon$ Eri, $\lambda$ And and V851 Cen, with $1 \sigma$ error bars. Solar coronal (at log $T[K] \sim 6.1$ ) to photospheric abundance ratios (Feldman \& Laming 2000) are indicated with a dotted line in the Procyon plot.

Photospheric abundances of different elements have been determined by many authors, resulting in a metallicity close to solar. In this work we will use the values calculated by Edvardsson et al. (1993), with C abundance from Varenne \& Monier (1999). First studies on the coronal abundances were carried out using EUVE observations: Drake et al. (1995) calculated, using a line-based analysis, the EMD for low and high FIP elements separately, but they did not find any "FIP" or "inverse FIP" effect. Global fits to Chandra/LETG and XMM-Newton high-resolution spectra were employed by Raassen et al. (2002) to determine the abundances of Procyon, also concluding that no FIP-related effects are present in the corona of Procyon (although no comparison with photospheric values is present in their study).

In the present work line fluxes were corrected from the ISM assuming a value of $\log N_{\mathrm{H}}\left(\mathrm{cm}^{-2}\right) \sim 18.2$ (Sanz-Forcada et al. 2003a). Several problems affect the analysis of the Chandra Procyon spectra. First, the shape of the continuum of a relatively cool corona like that of Procyon, in the wavelength range covered here, does not allow us to establish the $[\mathrm{Fe} / \mathrm{H}]$ coronal abundance accurately (a wide range of values show similar line-to-continuum ratios), thus we fixed it at its photospheric value (note that the rest of coronal abundances are calculated relative to $\mathrm{Fe}$, and the general level of the EMD depends on $[\mathrm{Fe} / \mathrm{H}]$ too). Also, the determination of coronal abundances is affected, in the LETG wavelength range, by the lack of a complete temperature coverage provided by Fe (only two lines are formed at $\log T[K] \lesssim 6.2$ ), while many lines from other elements have little temperature overlap with Fe lines. However it is possible to include in the analysis the Fe lines observed with EUVE (Fe XI-XVI), obtaining a better temperature coverage for one element, useful for the determination of abundances of other elements. Measurements of EUVE Fe line fluxes in the range 170-370 ̊ were made by Sanz-Forcada et al. (2003a) from observations taken in 1993, 1994 and 1999 (the latter is partially simultaneous with the LETG campaign, and no substantial changes were detected in the EUV flux level). The inclusion of the EUVE lines allows us to cover the range $\log T(K) \sim 5.6-6.8$, where almost all lines of other elements are also formed.

The abundances determined for Procyon do not show substantial differences between photospheric and coronal relative abundances (note that no error bars are published for the photospheric abundances), with the most notable difference being a relative underabundance of $\mathrm{O}$ in the corona. Nevertheless, an interesting feature arises when fitting the EMD and abundances. Assuming that the EMD (see Fig. 4) is correct for all elements, higher (relative to $\mathrm{Fe}$ ) abundance of $\mathrm{S}, \mathrm{O}, \mathrm{Ne}, \mathrm{Si}$ and $\mathrm{Mg}$ at lower temperatures would improve the fit of their respective lines (the case of the S VII lines is very remarkable), 

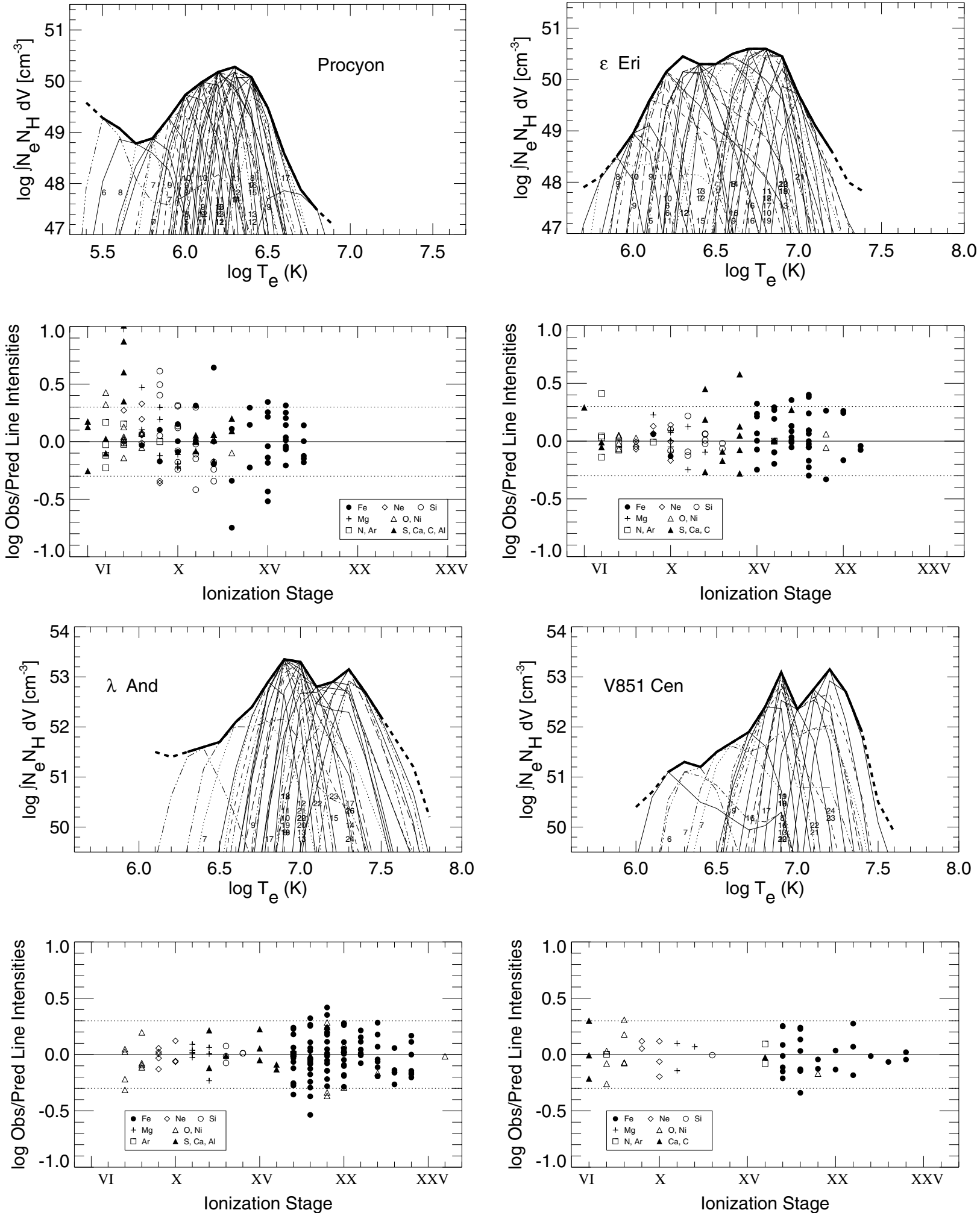

Fig. 4. Emission Measure Distribution for Procyon, $\epsilon$ Eri, $\lambda$ And and V851 Cen. Thin lines represent the relative contribution function for each ion (the emissivity function multiplied by the EMD at each point). Small numbers indicate the ionization stages of the species. Also plotted are the observed to predicted line flux ratios for the ion stages in the upper figure. The dotted lines denote a factor of 2.

while elements like $\mathrm{Ni}, \mathrm{Ca}, \mathrm{Al}, \mathrm{C}$ or $\mathrm{N}$ cover a gradient of temperatures too small to reveal any trend. This effect could be also caused by the Fe abundance increasing with temperature, from the minimum of the EMD at $\log T(K) \sim 5.8$ to the maximum at $\log T(K) \sim 6.3$. This might indicate a trend of a mild FIP effect which increases with temperature in the corona of Procyon (Drake et al. 1995 also discuss a possible temperature dependence of the coronal abundances in Procyon). In the 
Table 2. Coronal and photospheric abundances of the elements $([\mathrm{X} / \mathrm{H}]$, solar units) in the target stars.

\begin{tabular}{lrrrrrrrrrr}
\hline \hline $\mathrm{X}$ & \multicolumn{2}{c}{$\begin{array}{c}\text { FIP Reference } \\
\end{array}$} & \multicolumn{2}{c}{ Procyon } & \multicolumn{2}{c}{$\epsilon$ Eri } & \multicolumn{2}{c}{$\lambda$ And } & \multicolumn{2}{c}{$\begin{array}{c}\text { V851 Cen } \\
\text { CV }\end{array}$} \\
& solar value Photosphere & Corona $^{b}$ & Photosphere & Corona & Photosphere & Corona & Photosphere & Corona \\
\hline $\mathrm{Al}$ & 5.98 & 6.47 & 0.00 & $0.45 \pm 0.12$ & $-0.12 \pm 0.02$ & $\ldots$ & $\ldots$ & $-0.05 \pm 0.17$ & $0.25 \pm 0.05$ & \\
$\mathrm{Ca}$ & 6.11 & 6.36 & -0.04 & $-0.20 \pm 0.20$ & $-0.11 \pm 0.03$ & $0.23 \pm 0.20$ & $-0.20 \pm 0.10$ & $-0.25 \pm 0.37$ & $0.12 \pm 0.08$ & $0.50 \pm 0.47$ \\
$\mathrm{Ni}$ & 7.63 & 6.25 & 0.02 & $0.06 \pm 0.08$ & $-0.20 \pm 0.03$ & $-0.01 \pm 0.11$ & $-0.40 \pm 0.10$ & $-0.30 \pm 0.13$ & $-0.30 \pm 0.10$ & $0.10 \pm 0.44$ \\
$\mathrm{Mg}$ & 7.64 & 7.58 & 0.07 & $0.21 \pm 0.11$ & $-0.14 \pm 0.05$ & $-0.15 \pm 0.10$ & $-0.10 \pm 0.10$ & $-0.23 \pm 0.07$ & $0.05 \pm 0.03$ & $-0.12 \pm 0.17$ \\
$\mathrm{Fe}$ & 7.87 & 7.50 & -0.18 & $-0.18 \pm 0.06$ & $-0.12 \pm 0.01$ & $-0.30 \pm 0.20$ & $-0.50 \pm 0.10$ & $-0.60 \pm 0.05$ & $-0.23 \pm 0.10$ & $-0.50 \pm 0.10$ \\
$\mathrm{Si}$ & 8.15 & 7.55 & 0.01 & $0.06 \pm 0.10$ & $-0.16 \pm 0.02$ & $-0.21 \pm 0.07$ & $-0.30 \pm 0.10$ & $-0.39 \pm 0.07$ & $-0.05 \pm 0.09$ & $-0.62 \pm 0.32$ \\
$\mathrm{~S}$ & 10.36 & 7.21 & $\ldots$ & $-0.23 \pm 0.17$ & $-0.01 \pm 0.01$ & $0.00 \pm 0.15$ & $\ldots$ & $-0.67 \pm 0.16$ & $\ldots$ & $-1.05 \pm 1.33$ \\
$\mathrm{C}$ & 11.26 & 8.56 & 0.11 & $-0.10 \pm 0.14$ & -0.06 & $-0.28 \pm 0.18$ & $\ldots$ & $\ldots$ & $\ldots$ & $-0.37 \pm 0.40$ \\
$\mathrm{O}$ & 13.61 & 8.93 & -0.05 & $-0.44 \pm 0.10$ & $-0.16 \pm 0.02$ & $-0.40 \pm 0.04$ & $-0.25 \pm 0.10$ & $-0.30 \pm 0.13$ & $\ldots$ & $-0.09 \pm 0.23$ \\
$\mathrm{~N}$ & 14.53 & 8.05 & $\ldots$ & $-0.25 \pm 0.13$ & $\ldots$ & $-0.31 \pm 0.14$ & $\ldots$ & $\ldots$ & $\ldots$ & $0.00 \pm 0.14$ \\
$\mathrm{Ar}$ & 15.76 & 6.56 & $\ldots$ & $-0.43 \pm 0.13$ & $\ldots$ & $0.13 \pm 0.14$ & $\ldots$ & $-0.28 \pm 0.26$ & $\ldots$ & $-0.04 \pm 0.54$ \\
$\mathrm{Ne}$ & 21.56 & 8.09 & $\ldots$ & $0.02 \pm 0.11$ & $\ldots$ & $0.00 \pm 0.08$ & $\ldots$ & $-0.08 \pm 0.06$ & $\ldots$ & $0.41 \pm 0.13$ \\
\hline
\end{tabular}

${ }^{a}$ Solar photospheric abundances from Anders \& Grevesse (1989), adopted in this work, are expressed in logarithmic scale. Note that several values have been updated in the literature, most notably the cases of O (now 8.7, Allende Prieto et al. 2001; Holweger 2001) and C (now 8.39, Allende Prieto et al. 2002).

${ }^{b} \mathrm{The}[\mathrm{Fe} / \mathrm{H}]$ coronal abundance of Procyon is set to its photospheric value, with nominal error bars determined from the observed-to-predicted fluxes dispersion.

solar corona, the FIP effect is indeed larger at $\log T(K) \sim 6.15$ than at $\log T(K) \lesssim 5.9$ (Feldman \& Laming 2000). Although suggestive, this hypothesis cannot presently be confirmed with high confidence for Procyon given the present uncertainties in atomic models and in the determination of the EMD of this star. Similarly, an increase in the $[\mathrm{Ne} / \mathrm{Fe}]$ abundance with temperature between $\log T(K) \sim 5.8$ and $\log T(K) \sim 6.6$ would explain the discrepancies found in the determination of the coronal Ne abundance of AD Leo from Ne VIII lines (Sanz-Forcada $\&$ Micela 2002) and from Ne IX and Ne X lines (Maggio et al. 2002).

\section{2. $\epsilon$ Eri}

As one of the nearest cool stars $(d=3.22 \mathrm{pc}), \epsilon \operatorname{Eri}(\mathrm{HD} 22049$, HR 1084) is frequently studied for its activity, abundances, and more recently, stellar planetary companions (see e.g., Hatzes et al. 2000). With $P_{\text {phot }} \sim 11.3 \mathrm{~d}$ (Baliunas et al. 1983), $\epsilon$ Eri $(\mathrm{K} 2 \mathrm{~V})$ has an intermediate activity level. Photospheric abundances derived by Zhao et al. (2002) are used here for comparison with the coronal values, and a value of $\log N_{\mathrm{H}}\left(\mathrm{cm}^{-2}\right) \sim$ 18.1 (Sanz-Forcada et al. 2003a) is adopted to correct for ISM absorption. The first high-resolution analysis on coronal abundances of $\epsilon$ Eri was made by Laming et al. (1996) who analyzed EUVE spectra, calculating separate line-based EMDs for low- and high-FIP elements, concluding that a solar-like FIP effect was present in the corona of $\epsilon$ Eri. Coronal electron density diagnostics were reported by Ness et al. (2002) from the Chandra/LETG observations of $\epsilon$ Eri.

In the present work we derive the coronal abundance of 11 elements from the Chandra/LETG spectrum of $\epsilon$ Eri. The corona is dominated by emitting material in the range $\log T(K) \sim 5.8-7.2$, with a distribution compatible with the presence of a peak at $\log T(K) \sim 6.5$ as EUVE data suggest (Sanz-Forcada et al. 2003a), but also with two peaks at $\log T(K) \sim 6.3$ and 6.8, as the present data, also compatible with EUVE fluxes, suggest. The $[\mathrm{Fe} / \mathrm{H}]$ coronal abundance is better constrained than for Procyon, with a 0.2 dex uncertainty; the agreement between predicted and observed fluxes is also better than for Procyon. A mild FIP-like bias is detected in the corona of $\epsilon$ Eri, affecting only $\mathrm{Ca}$ and Ni (Fig. 3), with a progressive lower coronal abundance for elements with higher FIP - while in the Sun the FIP effect consists of a enhancement by more than a factor of 3 of the elements with FIP $\lesssim 10 \mathrm{eV}$.

\section{3. $\lambda$ And}

$\lambda$ And (HD 222107, HR 8961) is an active binary system with a G8 IV-III primary and an unseen companion in an orbit with $P_{\text {orb }}=20.5212 \mathrm{~d}$ (Walker 1944). $\lambda$ And is one of the few active stars for which measurements of photospheric abundances for a good number of elements (Savanov \& Berdyugina 1994; Donati et al. 1995) are available, also thanks to its low rotation rate $\left(P_{\text {phot }}=54.33 \mathrm{~d}\right.$, Perryman et al. 1997). Coronal abundances from high resolution spectra were not obtained from EUVE data because the ISM absorption did not allow the measurement of fluxes of lines for elements other than $\mathrm{Fe}$ (although the spectrum is suggestive of a low value of $[\mathrm{Fe} / \mathrm{H}]$, see Sanz-Forcada et al. 2001), and the global fit of the XMM/RGS spectra gave no clear trend with FIP (Audard et al. 2003). A preliminary analysis of the Chandra/HETG and LETG spectra was reported by Dupree et al. (2000) and Brickhouse et al. (2003) respectively.

The low ISM absorption ${ }^{2}\left(\log N_{\mathrm{H}}\left[\mathrm{cm}^{-2}\right]=18.45\right.$, Wood et al. 1996) makes the correction of fluxes unnecessary (affecting the line fluxes at less than the $1 \%$ level). The high

\footnotetext{
2 Note that this value of the ISM absorption, combined with the distance to the star, is too high to see lines from elements other than Fe that were measured for other stars (usually at $\lambda \gtrsim 200 \AA$ ) with EUVE, but it is a low value to notice its effects in the HETG spectral range.
} 
Table 3. Emission Measure Distribution of the target stars.

\begin{tabular}{|c|c|c|c|c|}
\hline \multirow{2}{*}{$\begin{array}{l}\log T \\
(\mathrm{~K})\end{array}$} & \multicolumn{4}{|c|}{$\overline{l o g} \int N_{\mathrm{e}} N_{\mathrm{H}} \mathrm{d} V\left(\mathrm{~cm}^{-3}\right)^{a}$} \\
\hline & Procyon & $\epsilon$ Eri & $\lambda$ And & V851 Cen \\
\hline 5.4 & 49.58 & & & \\
\hline 5.5 & $49.28_{-0.05}^{+0.55}$ & $\cdots$ & . & \\
\hline 5.6 & $49.08_{-0.30}^{+0.40}$ & & & \\
\hline 5.7 & $48.78_{-0.40}^{+0.20}$ & 47.60 & $\ldots$ & \\
\hline 5.8 & $48.88_{-0.40}^{+0.20}$ & 47.80 & $\ldots$ & \\
\hline 5.9 & $49.28_{-0.20}^{+0.20}$ & $48.20_{-0.30}^{+0.20}$ & .. & \\
\hline 6.0 & $49.73_{-0.40}^{+0.05}$ & $48.65_{-0.30}^{+0.20}$ & & 50.40 \\
\hline 6.1 & $49.98_{-0.05}^{+0.40}$ & $49.30_{-0.20}^{+0.20}$ & 50.90 & 50.70 \\
\hline 6.2 & $50.18_{-0.20}^{+0.05}$ & $49.85_{-0.20}^{+0.10}$ & 50.80 & $51.10_{-0.40}^{+0.05}$ \\
\hline 6.3 & $50.28_{-0.10}^{+0.05}$ & $50.15_{-0.20}^{+0.10}$ & $50.90_{-0.30}^{+0.20}$ & $51.30_{-0.35}^{+0.05}$ \\
\hline 6.4 & $50.08_{-0.05}^{+0.10}$ & $50.00_{-0.10}^{+0.20}$ & $51.00_{-0.30}^{+0.20}$ & $51.20_{-0.35}^{+0.05}$ \\
\hline 6.5 & $49.48_{-0.30}^{+0.05}$ & $50.00_{-0.30}^{+0.20}$ & $51.10_{-0.30}^{+0.20}$ & $51.50_{-0.40}^{+0.10}$ \\
\hline 6.6 & $48.58_{-0.40}^{+0.20}$ & $50.20_{-0.20}^{+0.20}$ & $51.50_{-0.40}^{+0.10}$ & $51.70_{-0.40}^{+0.20}$ \\
\hline 6.7 & $47.88_{-0.40}^{+0.30}$ & $50.30_{-0.25}^{+0.15}$ & $51.80_{-0.35}^{+0.05}$ & $51.90_{-0.35}^{+0.05}$ \\
\hline 6.8 & $47.48_{-0.40}^{+0.30}$ & $50.30_{-0.35}^{+0.05}$ & $52.30_{-0.15}^{+0.05}$ & $52.40_{-0.30}^{+0.05}$ \\
\hline 6.9 & 47.18 & $50.15_{-0.05}^{+0.15}$ & $52.75_{-0.05}^{+0.05}$ & $53.09_{-0.05}^{+0.05}$ \\
\hline 7.0 & $\ldots$ & $49.40_{-0.10}^{+0.40}$ & $52.70_{-0.05}^{+0.15}$ & $52.35_{-0.30}^{+0.20}$ \\
\hline 7.1 & . & $48.80_{-0.30}^{+0.40}$ & $52.20_{-0.20}^{+0.05}$ & $52.75_{-0.20}^{+0.20}$ \\
\hline 7.2 & .. & $48.30_{-0.30}^{+0.30}$ & $52.30_{-0.40}^{+0.05}$ & $53.15_{-0.30}^{+0.05}$ \\
\hline 7.3 & $\ldots$ & 47.70 & $52.55_{-0.40}^{+0.05}$ & $52.70_{-0.20}^{+0.30}$ \\
\hline 7.4 & $\ldots$ & 47.50 & $52.10_{-0.30}^{+0.20}$ & $51.90_{-0.40}^{+0.40}$ \\
\hline 7.5 & $\ldots$ & & $51.60_{-0.30}^{+0.40}$ & 50.50 \\
\hline 7.6 & $\ldots$ & 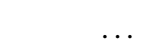 & 51.10 & 50.50 \\
\hline 7.7 & $\ldots$ & & 50.60 & \\
\hline 7.8 & $\ldots$ & $\cdots$ & 49.60 & \\
\hline
\end{tabular}

${ }^{a}$ Emission Measure, where $N_{\mathrm{e}}$ and $N_{\mathrm{H}}$ are electron and hydrogen densities, in $\mathrm{cm}^{-3}$. Error bars provided are not independent between the different temperatures, see text.

spectral resolution and good statistics achieved in the observation allow us to obtain the best determination of the element abundances of the four stars, also supported by a good agreement between observed and predicted fluxes for most lines. The fluxes measured in the EUV range (Sanz-Forcada et al. 2001) are also well predicted using the EMD determined here. The coronal abundances determined here are remarkably consistent with the photospheric values calculated by Donati et al. (1995), and they are also close to the values calculated by Savanov \& Berdyugina (1994).

\subsection{V851 Cen}

V851 Cen (HD 119285) is an active binary system with a K2IV-III primary and an unseen companion, with a photometric period of 12.05 d (Lloyd Evans \& Koen 1987; Cutispoto et al. 2001), slightly longer than its orbital period $\left(P_{\text {orb }}=\right.$ 11.989 d, Saar et al. 1990). V851 Cen has a small projected rotational velocity ( $v \sin i=6.5 \mathrm{~km} \mathrm{~s}^{-1}$, Saar et al. 1990) which allowed Katz et al. (2003) and Morel et al. (2003) to derive accurate photospheric abundances, revising the value of
$[\mathrm{Fe} / \mathrm{H}] \sim-0.6$ determined by Randich et al. (1993) to a value of $[\mathrm{Fe} / \mathrm{H}] \sim-0.2$. In the present work the coronal abundances and thermal structure of this star is determined for the first time. A correction of the observed fluxes due to the absorption of the ISM was applied, assuming $\log N_{\mathrm{H}}\left(\mathrm{cm}^{-2}\right) \sim 19.5$, the value determined for the nearby star $\beta$ Cen (Fruscione et al. $1994)^{3}$. We use the photospheric abundances provided by the "Method 1" of Morel et al. (2003), as shown in Fig. 2. When the coronal abundances are compared to solar photospheric ones, V851 Cen shows a behavior very similar to that of stars like AR Lac or AB Dor, with a decrease of abundance with FIP until $\mathrm{Si}$, and a progressive increment for elements with high FIP. However, when these abundances are compared with the stellar photospheric values, the conclusions are different, with only a small depletion of coronal $\mathrm{Fe}, \mathrm{Mg}$ and $\mathrm{Si}$, and a possible enhancement of the elements with lowest FIP (Ca and Ni), although still consistent with the photospheric values (Fig. 3). The determination of the photospheric abundance of elements with larger FIP would be desirable to confirm any trend.

\section{Discussion and conclusions}

The results found in the present sample show that the issue of coronal abundances in stars other than the Sun is complex and still poorly understood. Solar coronal abundance is by itself a complex issue, with the actual values depending on which part of the corona is observed (with coronal holes, active regions and flares showing quite different abundance patterns). Results obtained from full disk spectra (thus comparable to stellar spectra) show enhancements by at least a factor of 3 in elements with FIP $\lesssim 10 \mathrm{eV}$ (Laming et al. 1995). Young solar active regions show coronal abundance compatible with the photospheric values, but they soon develop a FIP bias that reaches average coronal values in two to three days (Sheeley 1996; Widing \& Feldman 2001). The FIP effect that is observed in the solar corona seems to disappear with increasing activity level of the star, as it has been shown in cases like II Peg (Huenemoerder et al. 2001), AR Lac (Huenemoerder et al. 2003), AB Dor (Sanz-Forcada et al. 2003b), $\lambda$ And and V851 Cen. A solar-like FIP effect appears to be present in $\alpha$ Cen A (Raassen et al. 2003), a star very similar to the Sun in many physical parameters, as well as in other solar analogs with low levels of activity, such as $\pi^{1}$ UMa and $\chi^{1}$ Ori (Güdel et al. 2002), although only the Fe photospheric abundance is known for these two objects (so that abundance patterns assume a solar-like mix). A mid-activity level star, $\epsilon$ Eri, could be showing the progressive disappearance of the FIP effect, with elements like $\mathrm{Mg}$ and $\mathrm{Fe}$ already showing photospheric abundances in the corona, and leaving a marginal coronal abundance enhancement only for $\mathrm{Ca}$ and Ni. Finally, Procyon seems to be quite different to the Sun, with no FIP-related trends evident, although there could be an increment of Fe abundance with the coronal temperature relative to other elements with higher FIP (see Sect. 4.1). The FIP effect detected in the solar

\footnotetext{
3 Uncertainties in the determination of the ISM absorption are of minor importance given the small correction (at the $\sim 2 \%$ level at $20 \AA$ ) to be applied to the line fluxes.
} 
corona above quiet regions grows with temperature, in a range similar to that covered here for Procyon (Feldman \& Laming 2000).

The interpretation of the metal depletion in the corona of active stars that has been given in the past from initial results is somewhat in contradiction with the present results. Many of the stars for which a "metal abundance deficiency syndrome" (MAD) has been claimed do not have measurements of the photospheric abundance available (or they are limited to Fe abundance determinations), and published work is limited to the comparison of stellar coronal patterns with solar photospheric abundances (a rather arbitrary practice). The case of $\lambda$ And is quite remarkable, with compatible coronal and photospheric abundances for all elements tested, and a definitely non-solar photospheric abundance pattern. V851 Cen, with a similar coronal thermal structure (Fig. 4) shows a marginal coronal abundance depletion. Other cases like II Peg (Huenemoerder et al. 2001) or AR Lac (Huenemoerder et al. 2003) also show moderate depletion when they are compared to the respective photospheric values, but strong metal depletion seems to be clear only for AB Dor (Sanz-Forcada et al. 2003b, and references therein). However AB Dor is a fast rotator, and the only available determination of photospheric abundances, carried out by Vilhu et al. (1987), needs further confirmation given the difficulty of the measurements in a spectrum so highly affected by rotational broadening.

While the absence of a FIP effect in active stars, at least at the solar level, is well supported at this point, the presence of a general "MAD effect" or an "inverse FIP effect" increasing with activity is not yet clearly established, given the discrepancies found between stars with quite similar levels of activity and thermal coronal structure like AB Dor, $\lambda$ And and V851 Cen. Moreover, the abundance pattern could also be changing in different parts of the corona, depending on the coronal temperature, as present in Procyon. A larger sample of stars with both photospheric and coronal abundances, determined in a homogeneous way, would be necessary to establish a general trend between coronal abundances and activity levels.

Acknowledgements. We acknowledge support by the Marie Curie Fellowships Contract No. HPMD-CT-2000-00013. We have made use of data obtained through the Chandra data archive, operated by the Smithsonian Astrophysical Observatory for NASA. This research has also made use of NASA's Astrophysics Data System Abstract Service. We are grateful to the referee, Dr. J. M. Laming, for the careful reading and useful comments brought to the manuscript.

\section{References}

Allende Prieto, C., Lambert, D. L., \& Asplund, M. 2001, ApJ, 556, L63

Allende Prieto, C., Lambert, D. L., \& Asplund, M. 2002, ApJ, 573, L137

Anders, E., \& Grevesse, N. 1989, Geochim. Cosmochim. Acta, 53, 197

Audard, M., Güdel, M., Sres, A., Raassen, A. J. J., \& Mewe, R. 2003, A\&A, 398, 1137

Baliunas, S. L., Hartmann, L., Noyes, R. W., et al. 1983, ApJ, 275, 752
Brickhouse, N. S., Dupree, A. K., \& Hoogerwerf, R. 2003, AAS/High Energy Astrophysics Division, 35

Brinkman, A. C., Behar, E., Güdel, M., et al. 2001, A\&A, 365, L324

Cayrel de Strobel, G., Soubiran, C., \& Ralite, N. 2001, A\&A, 373, 159

Cutispoto, G., Messina, S., \& Rodonò, M. 2001, A\&A, 367, 910

de Medeiros, J. R., \& Mayor, M. 1999, A\&AS, 139, 433

den Herder, J. W., Brinkman, A. C., Kahn, S. M., et al. 2001, A\&A, 365, L7

Donati, J.-F., Henry, G. W., \& Hall, D. S. 1995, A\&A, 293, 107

Drake, J. J. 2002, in Stellar Coronae in the Chandra and XMM-Newton Era, ASP Conf. Ser., 277, 75

Drake, J. J., Brickhouse, N. S., Kashyap, V., et al. 2001, ApJ, 548, L81

Drake, J. J., Laming, J. M., \& Widing, K. G. 1995, ApJ, 443, 393

Drake, J. J., Laming, J. M., \& Widing, K. G. 1997, ApJ, 478, 403

Drake, S. A. 1996, in Cosmic Abundances, ed. S. S. Holt, \& G. Sonneborn, ASP Conf. Ser., 99, 215

Dupree, A. K., Brickhouse, N. S., \& Drake, J. J. 2000, Bull. Am. Astron. Soc., 32, 1256

Edvardsson, B., Andersen, J., Gustafsson, B., et al. 1993, A\&A, 275, 101

Favata, F., \& Micela, G. 2003, Space Sci. Rev., 108, 577

Feldman, U., \& Laming, J. M. 2000, Phys. Scr., 61, 222

Fruscione, A., Hawkins, I., Jelinsky, P., \& Wiercigroch, A. 1994, ApJS, 94, 127

Güdel, M., Audard, M., Sres, A., et al. 2002, in Stellar Coronae in the Chandra and XMM-Newton Era, ASP Conf. Ser., 277, 497

Hatzes, A. P., Cochran, W. D., McArthur, B., et al. 2000, ApJ, 544, L145

Holweger, H. 2001, in Joint SOHO/ACE workshop Solar and Galactic Composition, ed. R. F. Wimmer-Schweingruber (New York: Springer), AIP Conf. Proc., 598, 23

Houck, J. C., \& Denicola, L. A. 2000, in Astronomical Data Analysis Software and Systems IX, ed. N. Manset, C. Veillet, \& D. Crabtree (San Francisco: ASP), ASP Conf. Ser., 216, 591

Huenemoerder, D. P., Canizares, C. R., Drake, J. J., \& Sanz-Forcada, J. 2003, ApJ, 595, 1131

Huenemoerder, D. P., Canizares, C. R., \& Schulz, N. S. 2001, ApJ, 559,1135

Irwin, A. W., Fletcher, J. M., Yang, S. L. S., Walker, G. A. H., \& Goodenough, C. 1992, PASP, 104, 489

Katz, D., Favata, F., Aigrain, S., \& Micela, G. 2003, A\&A, 397, 747

Kelly, R. L. 1987, Atomic and ionic spectrum lines below 2000 Angstroms. Hydrogen through Krypton (New York: American Institute of Physics, Amer. Chem. Soc. and the Nat. Bureau of Standards)

Laming, J. M., \& Drake, J. J. 1999, ApJ, 516, 324

Laming, J. M., Drake, J. J., \& Widing, K. G. 1995, ApJ, 443, 416

Laming, J. M., Drake, J. J., \& Widing, K. G. 1996, ApJ, 462, 948

Lloyd Evans, T., \& Koen, M. C. J. 1987, South African Astron. Obs. Circ., 11, 21

Maggio, A., Drake, J. J., Kashyap, V., et al. 2002, in Stellar Coronae in the Chandra and XMM-Newton Era, ASP Conf. Ser., 277, 57

Morel, T., Micela, G., Favata, F., Katz, D., \& Pillitteri, I. 2003, A\&A, 412, 495

Ness, J.-U., Schmitt, J. H. M. M., Burwitz, V., et al. 2002, A\&A, 394, 911

Perryman, M. A. C., Lindegren, L., Kovalevsky, J., et al. 1997, A\&A, 323, L49

Raassen, A. J. J., Mewe, R., Audard, M., et al. 2002, A\&A, 389, 228

Raassen, A. J. J., Ness, J.-U., Mewe, R., et al. 2003, A\&A, 400, 671

Randich, S., Gratton, R., \& Pallavicini, R. 1993, A\&A, 273, 194

Saar, S. H., Nordstrom, B., \& Andersen, J. 1990, A\&A, 235, 291 
Sanz-Forcada, J., Brickhouse, N. S., \& Dupree, A. K. 2001, ApJ, 554, 1079

Sanz-Forcada, J., Brickhouse, N. S., \& Dupree, A. K. 2003a, ApJS, 145,147

Sanz-Forcada, J., Maggio, A., \& Micela, G. 2003b, A\&A, 408, 1087 Sanz-Forcada, J., \& Micela, G. 2002, A\&A, 394, 653

Savanov, I. S., \& Berdyugina, S. V. 1994, Astron. Lett., 20, 227

Sheeley, N. R. 1996, ApJ, 469, 423

Smith, R. K., Brickhouse, N. S., Liedahl, D. A., \& Raymond, J. C. 2001, ApJ, 556, L91
Varenne, O., \& Monier, R. 1999, A\&A, 351, 247

Vilhu, O., Gustafsson, B., \& Edvardsson, B. 1987, ApJ, 320, 850

Walker, E. C. 1944, JRASC, 38, 249

Weisskopf, M. C., Brinkman, B., Canizares, C., et al. 2002, PASP, 114,1

White, N. E., Arnaud, K., Day, C. S. R., et al. 1994, PASJ, 46, L97

Widing, K. G., \& Feldman, U. 2001, ApJ, 555, 426

Wood, B. E., Alexander, W. R., \& Linsky, J. L. 1996, ApJ, 470, 1157

Zhao, G., Chen, Y. Q., Qiu, H. M., \& Li, Z. W. 2002, AJ, 124, 2224 